\title{
Supersimplicity and countable reducts of a unidimensional hypersimple theory
}

\author{
Ziv Shami
}

September 3, 2018

\begin{abstract}
We show that a hypersimple unidimensional theory that has a club of reducts, in the partial order of all countable reducts, that are coordinatized in finite rank, is supersimple.
\end{abstract}

\section{Introduction}

In this paper we suggest an approach to the problem on supersimplicity of unidimensional hypersimple theories. The problem has been answered in the affirmative in the following cases. In $[\mathrm{H}]$, for any stable theory, in [S1] for any countable theory (this improved an earlier result for the case of a countable theory with the wnfcp $[\mathrm{P}]$ ) and in [S2] it has been proved for any (possibly uncountable) non-s-essentially 1-based theory (roughly, a theory that is far from being 1-based).

It is easy to see that supersimplicity of a theory is determined by (the supersimplicity of) the family of its countable reducts. Therefore, it is natural to try and reflect properties of the given unidimensional hypersimple theory to countable reducts. Clearly, unidimensionality is not preserved under reducts. On the other hand, easily any unidimensional hypersimple theory is coordinatized in finite rank (see Definition 5.1). In this paper we show that supersimplicity of any (possibly uncountable) unidimensional hypersimple theory follows from coordinatization in finite rank of sufficiently many countable reducts of it.

We thank Ehud Hrushovski for discussions on this topic and for allowing us to include a remark of him about elimination of hyperimaginaries in 
reducts (section 3). We will assume basic knowledge of simple theories as in $[\mathrm{K} 1],[\mathrm{KP}],[\mathrm{HKP}]$. A good text book on simple theories that covers much more is $[\mathrm{W}]$. The notations are standard, and throughout the paper we work in a large saturated model $\mathcal{C}$ of a complete first-order theory $T$ in a language $L$.

\section{Preliminaries}

In this section $T$ is assumed to be simple. We quote several known facts that we will apply.

\subsection{Almost internality, analyzability and unidimension- ality}

In this subsection we work in $\mathcal{C}$ with hyperimaginaries unless otherwise stated; if $T$ is hypersimple (i.e. simple and eliminates hyperimaginaries) and we work in $\mathcal{C}^{e q}$ we get equivalent definitions.

In this subsection, $\mathcal{P}$ denotes an $A$-invariant family of partial types and $p$ a partial type over $A$. We say that $p$ is (almost-) $\mathcal{P}$-internal for every realization $a$ of $p$ there exists $b$ with $a \underset{A}{\downarrow} b$ such that for some tuple $c$ of realizations of partial types in $\mathcal{P}$ over $A b$ we have $a \in d c l(b, c)$ (respectively, $a \in \operatorname{acl}(b, c))$. We say that $p$ is analyzable in $\mathcal{P}$ if for any $a \models p$ there exists a sequence $I=\left\langle a_{i} \mid i \leq \alpha\right\rangle$ such that $a_{\alpha}=a$ and $t p\left(a_{i} /\left\{a_{j} \mid j<i\right\} \cup A\right)$ is almost $\mathcal{P}$-internal for every $i \leq \alpha$.

First, the following fact is straightforward.

Fact 2.1 1) Assume $t p\left(a_{i} / A\right)$ are (almost) $\mathcal{P}$-internal for $i<\alpha$. Then $t p\left(\left\langle a_{i} \mid i<\alpha\right\rangle / A\right)$ is (respectively, almost) $\mathcal{P}$-internal. Thus, if $t p\left(a_{i} / A\right)$ are analyzable in $\mathcal{P}$ for $i<\alpha$. Then $\operatorname{tp}\left(\left\langle a_{i} \mid i<\alpha\right\rangle / A\right)$ is analyzable in $\mathcal{P}$.

2) If $\operatorname{tp}(a / A)$ almost $\mathcal{P}$-internal, so is $\operatorname{tp}(a / B)$ for any set $B \supseteq A$.

$T$ is said to be unidimensional if whenever $p$ and $q$ are complete non-algebraic types, $p, q$ are non-orthogonal.

We will also need the following easy Fact. 
Fact 2.2 Work in $\mathcal{C}$ (without hyperimaginaries). Let $p \in S(\emptyset)$ and let $\theta \in L$. Assume $p$ is analyzable in $\theta$. Then $p$ is analyzable in $\theta$ in finitely many steps. In particular, if $T$ is a hypersimple unidimensional theory and there exists a non-algebraic supersimple definable set, then $T$ has finite $S U$-rank, i.e. every complete type has finite $S U$-rank. In fact, for every given sort there is a finite bound on the $S U$-rank of all types in that sort, equivalently the global D-rank of any sort is finite.

Another useful fact is the following.

Fact $2.3[S 1]$ Let $T$ be any unidimensional simple theory. Then $T$ eliminates $\exists^{\infty}$.

\subsection{The forking topology, EPFO and PCFT}

The forking topology is introduced in [S0] and is a variant of Hrushovski's and Pillay's topologies from $[\mathrm{H} 0]$ and $[\mathrm{P}]$, respectively. In this section $T$ is assumed to be simple and we work in $\mathcal{C}$.

Definition 2.4 Let $A \subseteq \mathcal{C}$ and let $x$ be a finite tuple of variables.

1) An invariant set $\mathcal{U}$ over $A$ is said to be a basic $\tau^{f}$-open set over $A$ if there is $\phi(x, y) \in L(A)$ such that

$$
\mathcal{U}=\{a \mid \phi(a, y) \text { forks over } A\} .
$$

Note that the family of basic $\tau^{f}$-open sets over $A$ is closed under finite intersections, thus forms a basis for a unique topology on $S_{x}(A)$. An open set in this topology is called a $\tau^{f}$-open set over $A$ or a forking-open set over $A$.

2) An invariant set $\mathcal{U}$ over $A$ is said to be a basic $\tau_{\infty}^{f}$-open set over $A$ if $\mathcal{U}$ is a type-definable $\tau^{f}$-open set over $A$. The family of basic $\tau_{\infty}^{f}$-open sets over $A$ is a basis for a unique topology on $S_{x}(A)$. An open set in this topology is called a $\tau_{\infty}^{f}$-open set over $A$.

Remark 2.5 The $\tau_{\infty}^{f}$-topology and in particular the $\tau^{f}$-topology on $S_{x}(A)$ refines the Stone-topology of $S_{x}(A)$ for all $x, A$.

We will apply the following Fact.

Fact 2.6 Let $\mathcal{U}$ be a $\tau^{f}$-open set over $\emptyset$ and let $A$ be any set. Then $\mathcal{U}$ is $\tau^{f}$-open over $A$. 
Recall the following definition from [S0].

Definition 2.7 We say that the $\tau^{f}$-topologies over $A$ are closed under projections ( $T$ is PCFT over $A$ ) if for every $\tau^{f}$-open set $\mathcal{U}(x, y)$ over $A$ the set $\exists y \mathcal{U}(x, y)$ is a $\tau^{f}$-open set over $A$. We say that the $\tau^{f}$-topologies are closed under projections ( $T$ is PCFT) if they are such over every set $A$.

In [BPV, Proposition 4.5] the authors proved the following equivalence which, for convenience, we will use as a definition (their definition involves extension with respect to pairs of models of $T$ ).

Definition 2.8 We say that the extension property is first-order in $T$ or $T$ is EPFO iff for every formulas $\phi(x, y), \psi(y, z) \in L$ the relation $Q_{\phi, \psi}$ defined by:

$$
Q_{\phi, \psi}(a) \text { iff } \phi(x, b) \text { doesn't fork over } a \text { for every } b \models \psi(y, a)
$$

is type-definable (here $a$ can be an infinite tuple from $\mathcal{C}$ whose sorts are fixed).

Fact $2.9[S 1$, Corollary 3.13] Suppose the extension property is first-order in T. Then T is PCFT.

We say that an $A$-invariant set $\mathcal{U}$ has finite $S U$-rank if $S U(a / A)<\omega$ for all $a \in \mathcal{U}$, and has bounded finite $S U$-rank if there exists $n<\omega$ such that $S U(a / A) \leq n$ for all $a \in \mathcal{U}$. The existence of a $\tau^{f}$-open set of bounded finite $S U$-rank implies the existence of an $S U$-rank 1 formula (i.e. a weaklyminimal formula):

Fact 2.10 [S0, Proposition 2.13] Let $\mathcal{U}$ be an unbounded $\tau^{f}$-open set over some set $A$. Assume $\mathcal{U}$ has bounded finite $S U$-rank. Then there exist a set $B \supseteq A$ with $|B \backslash A|<\omega$ and $\theta(x) \in L(B)$ of $S U$-rank 1 such that $\theta^{\mathcal{C}} \subseteq$ $\mathcal{U} \cup \operatorname{acl}(B)$.

Now, recall the following two facts and their corollary. First, let $\mathcal{P}^{S U \leq 1}$ denote the class of complete real types over sets of size $\leq|T|$, of $S U$-rank $\leq 1$

Fact $2.11[P 1]$ Let $T$ be a simple theory that eliminates $\exists^{\infty}$. Moreover, assume every type is analyzable in $\mathcal{P}^{S U \leq 1}$. Then the extension property is first-order in $T$.

For a more general statement, see [S1, Lemma 3.7]. 


\subsection{Stable independence and stable SU-rank}

In this subsection $T$ is assumed to be simple and we work in $\mathcal{C}$.

First we recall the notion of stable independence.

Definition 2.12 Let $a \in \mathcal{C}, A \subseteq B \subseteq \mathcal{C}$. We say that $a$ is stably-independent from $B$ over $A$ if for every stable $\phi(x, y) \in L$, if $\phi(x, b)$ is over $B$ and $a^{\prime} \models$ $\phi(x, b)$ for some $a^{\prime} \in d c l(A a)$, then $\phi(x, b)$ doesn't divide over $A$. In this case we denote it by $\begin{aligned} & \underset{S}{S} B . \\ & A\end{aligned}$.

The notion of stable $S U$-rank is defined via stable dependence.

Definition 2.13 1) For $a \in \mathcal{C}$ and $A \subseteq \mathcal{C}$ the $S U_{s}$-rank is defined by induction on $\alpha$ : if $\alpha=\beta+1$, then $S U_{s}(a / A) \geq \alpha$ if there exists $B \supseteq A$ such that $a \underset{A}{X_{s}} B$ and $S U_{s}(a / B) \geq \beta$. For limit $\alpha, S U_{s}(a / A) \geq \alpha$ if $S U_{s}(a / A) \geq \beta$ for all $\beta<\alpha$.

2) Let $\mathcal{U}$ be an $A$-invariant set. We write $S U_{s}(\mathcal{U})=\alpha$ (the $S U_{s}$-rank of $\mathcal{U}$ is $\alpha$ ) if $\operatorname{Max}\left\{S U_{s}(p) \mid p \in S(A), p^{\mathcal{C}} \subseteq \mathcal{U}\right\}=\alpha$. We say that $\mathcal{U}$ has bounded finite $S U_{s}$-rank if for some $n<\omega, S U_{s}(\mathcal{U})=n$. Note that the $S U_{s}$-rank of $\mathcal{U}$ might, a priori, depend on the choice of the set $A$ over which $\mathcal{U}$ is invariant.

The following rank is a variation of stable $S U$-rank; it is non-increasing in extensions.

Definition 2.14 1) For $a \in \mathcal{C}$ and $A \subseteq \mathcal{C}$ the $S U_{\text {se }}$-rank is defined by induction on $\alpha$ : if $\alpha=\beta+1, S U_{s e}(a / A) \geq \alpha$ if there exist $B_{1} \supseteq B_{0} \supseteq A$ such that $a \underset{B_{0}}{X_{0}} B_{1}$ and $S U_{s e}\left(a / B_{1}\right) \geq \beta$. For limit $\alpha, S U_{s e}(a / A) \geq \alpha$ if $S U_{\text {se }}(a / A) \geq \beta$ for all $\beta<\alpha$.

2) Let $\mathcal{U}$ be an $A$-invariant set. We write $S U_{s e}(\mathcal{U})=\alpha$ (the $S U_{s e}$-rank of $\mathcal{U}$ is $\alpha$ ) if $\operatorname{Max}\left\{S U_{s e}(p) \mid p \in S(A), p^{\mathcal{C}} \subseteq \mathcal{U}\right\}=\alpha$. We say that $\mathcal{U}$ has bounded finite $S U_{s e}$-rank if for some $n<\omega, S U_{s e}(\mathcal{U})=n$.

Remark 2.15 Note that $S U_{s e}(a / B) \leq S U_{s e}(a / A)$ for all $a \in \mathcal{C}$ and $A \subseteq$ $B \subseteq \mathcal{C}$ (this is the reason for introducing $\left.S U_{s e}\right)$. Also, clearly $S U_{s}(a / A) \leq$ $S U_{s e}(a / A) \leq S U(a / A)$ for all $a, A$. Clearly $S U_{s e}(a / A)=0$ iff $S U_{s}(a / A)=0$ iff $a \in \operatorname{acl}(A)$ for all $a, A$. 
We will apply the following easy fact.

Fact 2.16 For $a \in \mathcal{C}$ and $A \subseteq B \subseteq \mathcal{C}$, assume tp $(a / B)$ doesn't fork over

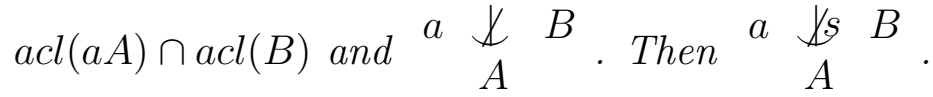

\section{Elimination of hyperimaginaries in reducts}

In this section we include a remark by Ehud Hrushovski that allowed us to remove the assumption that the reducts eliminate hyperimaginaries (in the main theorem). Here $T$ denotes any complete theory in a language $L$ and we work in $\mathcal{C}$.

Definition 3.1 A reduct $T^{-}$of $T$ to a sublanguage $L^{-} \subseteq L$ is said to be $E$-closed if for every $L^{-}$-definable sets $D_{1} \vdash D_{2}$ on $S^{2}$ (for some sort $S$ of $\left.L^{-}\right)$there exists a definable equivalence relation $E^{-} \in L^{-}$satisfying $D_{1} \vdash$ $E^{-} \vdash D_{2}$, provided that there exists such definable equivalence relation in $L$.

For a partial order $(P, \leq)$, a subset $A \subseteq P$ is called a club in $(P, \leq)$, if $A$ unbounded in $(P, \leq)$, that is, above any element of $P$ there is an element of $A$, and $A$ is closed in $(P, \leq)$, that is, for any chain $C \subseteq A$, if $a \in P$ is the supremum of $C$ (i.e. $a$ is an upper bound of $C$ and $a$ is smaller then any other upper bound of $C$ ) then $a \in A$.

Notation 3.2 Let $T^{-}$be a reduct of $T$ to $L^{-}$. The size of the reduct $T^{-}$ is just $\left|T^{-}\right|$. Let $\lambda$ be any infinite cardinal $($ or $\infty)$. Let $\left(\mathcal{R}_{T}^{\lambda}, \leq_{T}\right)$ be the partial order of all reducts of $T$ of size $\leq \lambda$, where the order is just inclusion (of the sublanguages of the reducts, i.e. of both the set of sorts and the set of formulas). It will be convenient to consider the isomorphic partial order $\left(\mathcal{R}_{\mathcal{C}}^{\lambda}, \leq_{\mathcal{C}}\right)$ of all the (saturated) model reducts of $\mathcal{C}$ to a sublanguage of $L$ size $\leq \lambda$.

Claim 3.3 Let $T$ be any complete L-theory that eliminates hyperimaginaries. 1) Let $T^{-}$be an E-closed reduct of $T$. Then $T^{-}$eliminates hyperimaginaries.

2) The set of E-closed reducts of $T$ is a club in $\left(\mathcal{R}_{T}^{\infty}, \leq_{T}\right)$. Given any infinite $\lambda \leq|L|$, the set of $E$-closed reducts of $T$ of size $\leq \lambda$ is a club in $\left(\mathcal{R}_{T}^{\lambda}, \leq_{T}\right)$. 
Proof: 1) Say $T^{-}$is the reduct of $T$ to $L^{-}$and so $\mathcal{C} \mid L^{-}$is a saturated model of $T^{-}$. We claim that the hyperimaginaries of $T^{-}$are eliminated, namely: for every type-definable equivalence relation $E^{-}$of $T^{-}$on a complete type $p^{-}$of $T^{-}$over $\emptyset$, there are definable equivalence relations $E_{i}^{-} \in L^{-}$such that $E^{-}$is equivalent to $\bigwedge_{i} E_{i}^{-}$on $p^{-}$. Indeed, let $E^{-}=E^{-}\left(x, x^{\prime}\right)$ and $p^{-}=$ $p^{-}(x)$ be such. Let $\phi_{i}^{-}\left(x, x^{\prime}\right) \in L^{-}$be such that $E^{-}\left(x, x^{\prime}\right)=\bigwedge_{i} \phi_{i}^{-}\left(x, x^{\prime}\right)$. Let $p$ be any complete type of $T$ over $\emptyset$ that extends $p^{-}$. By elimination of hyperimaginaries in $T$, there are $E_{j}\left(x, x^{\prime}\right) \in L$ such that $\Lambda_{j} E_{j}\left(x, x^{\prime}\right)$ is equivalent to $E^{-}\left(x, x^{\prime}\right)$ on $p^{\mathcal{C}}$. Now, by compactness, for any $i$ there is $j(i)$ such that $E_{j(i)}\left(x, x^{\prime}\right) \vdash \phi_{i}^{-}\left(x, x^{\prime}\right)$ on $p^{\mathcal{C}}$, likewise, for every $j$ there exists $k(j)$

such that $\phi_{k(j)}^{-}\left(x, x^{\prime}\right) \vdash E_{j}\left(x, x^{\prime}\right)$ on $p^{\mathcal{C}}$. As $T^{-}$is an $E$-closed reduct of $T$, for every $i$, there is a definable equivalence relation $E_{i}^{-} \in L^{-}$such that $\phi_{k(j(i))}^{-}\left(x, x^{\prime}\right) \vdash E_{i}^{-}\left(x, x^{\prime}\right) \vdash \phi_{i}^{-}\left(x, x^{\prime}\right)$ on $p^{\mathcal{C}}$ (using compactness). We conclude that $E^{-}$is equivalent to $\bigwedge_{i} E_{i}^{-}\left(x, x^{\prime}\right)$ on $p^{\mathcal{C}}$ and thus on $p^{-\mathcal{C}}$ as well (as $E^{-}$ and $E_{i}^{-}$are all invariant under automorphisms of $\left.\mathcal{C} \mid L^{-}\right)$. 2) is immediate.

\section{Dichotomies for $\emptyset$-invariant families of rank 1 types}

Here we verify the following extension of [S2, Corollary 2.13] to a general $\emptyset$-invariant family of $S U$-rank 1 types. In this section $T$ is assumed to be a simple theory with elimination of imaginaries.

We first recall some basic definitions from [S1].

Definition 4.1 A family

$$
\Upsilon=\left\{\Upsilon_{x, A} \mid x \text { is a finite sequence of variables and } A \subset \mathcal{C} \text { is small }\right\}
$$

is said to be a projection closed family of topologies if each $\Upsilon_{x, A}$ is a topology on $S_{x}(A)$ that refines the Stone-topology on $S_{x}(A)$, this family is invariant under automorphisms of $\mathcal{C}$ and change of variables by variables of the same sort, the family is closed under product by the full Stone spaces $S_{y}(A)$ (where $y$ is a disjoint tuple of variables) and closed by projections, namely whenever $\mathcal{U}(x, y) \in \Upsilon_{x y, A}, \exists y \mathcal{U}(x, y) \in \Upsilon_{x, A}$.

We will be interested in the case $\Upsilon=\tau^{f}$, where $T$ is a PCFT theory. From now on fix a general projection closed family $\Upsilon$ of topologies. 
Definition 4.21$)$ A type $p \in S(A)$ is said to be s-essentially 1-based over $A_{0} \subseteq A$ (essentially 1-based over $A_{0} \subseteq A$ ) by means of $\Upsilon$ if for every finite tuple $\bar{c}$ from $p$ and for every (respectively, type-definable) $\Upsilon$-open set $\mathcal{U}$ over $A \bar{c}$, with the property that $a$ is independent from $A$ over $A_{0}$ for every $a \in \mathcal{U}$, the set $\left\{a \in \mathcal{U} \mid C b(a / A \bar{c}) \notin b d d\left(a A_{0}\right)\right\}$ is nowhere dense in the Stone-topology of $\mathcal{U}$. We say $p \in S(A)$ is s-essentially 1-based (essentially 1-based) by means of $\Upsilon$ if $p$ is s-essentially 1-based (respectively, essentially 1-based) over $A$ by means of $\Upsilon$.

2) Let $V$ be an $A_{0}$-invariant set and let $p \in S\left(A_{0}\right)$. We say that $p$ is analyzable in $V$ by s-essentially 1-based (by essentially 1-based) types by means of $\Upsilon$ if there exists $a \models p$ and there exists a sequence $\left(a_{i} \mid i \leq \alpha\right) \subseteq \operatorname{dcl}\left(A_{0} a\right)$ with $a_{\alpha}=a$ such that $\operatorname{tp}\left(a_{i} / A_{0} \cup\left\{a_{j} \mid j<i\right\}\right)$ is $V$-internal and s-essentially 1-based (respectively, essentially 1-based) over $A_{0}$ by means of $\Upsilon$ for all $i \leq \alpha$.

Theorem 4.3 Let $T$ be any countable hypersimple theory with PCFT. Let $\mathcal{P}_{0}$ be an $\emptyset$-invariant family of $S U$-rank 1 partial types. Then, either there exists a weakly-minimal formula that is almost $\mathcal{P}_{0}$-internal, or every complete type $p \in S(A)$ that is internal in $\mathcal{P}_{0}$ is essentially 1-based over $\emptyset$ by means of $\tau^{f}$. In particular, either there exists a weakly-minimal formula that is almost $\mathcal{P}_{0}$-internal, or whenever $p \in S(A)$, where $A$ is countable, and $p$ is analyzable in $\mathcal{P}_{0}, p$ is analyzable in $\mathcal{P}_{0}$ by essentially 1-based types by means of $\tau^{f}$.

The most general dichotomy theorem of this type that we present is the following theorem that generalizes [S2, Theorem 2.3] to any $\emptyset$-invariant family of $S U$-rank 1 types. The proof of this theorem is almost identical to the proof of [S2, Theorem 2.3] (but note that the next version that we present with a proof contains all modifications that are needed for the proof of it).

Theorem 4.4 Let $T$ be any hypersimple theory. Let $\Upsilon$ be a projection-closed family of topologies. Let $\mathcal{P}_{0}$ be an $\emptyset$-invariant family of $S U$-rank 1 types. Then, either there exists an unbounded $\Upsilon$-open set (over some small set $A$ ) that is almost $\mathcal{P}_{0}$-internal (and in particular has finite $S U$-rank), or every complete type $p \in S(A)$ that is internal in $\mathcal{P}_{0}$ is s-essentially 1-based over $\emptyset$ by means of $\Upsilon$. In particular, either there exists an unbounded $\Upsilon$-open set that is almost $\mathcal{P}_{0}$-internal, or whenever $p \in S(A)$ and $p$ is analyzable in $\mathcal{P}_{0}$ , $p$ is analyzable in $\mathcal{P}_{0}$ by s-essentially 1-based types by means of $\Upsilon$.

The next theorem is a version of Theorem 4.4 for a countable language with a stronger consequence and is a generalization of [S2, Theorem 2.11] to 
$\emptyset$-invariant family of $S U$-rank 1 types. We give the complete proof of this theorem.

Theorem 4.5 Let $T$ be any countable hypersimple theory. Let $\Upsilon$ be a projectionclosed family of topologies such that $\left\{a \in \mathcal{C}^{x} \mid a \notin \operatorname{acl}(A)\right\} \in \Upsilon_{x, A}$ for all $x$ and set $A$. Let $\mathcal{P}_{0}$ be an $\emptyset$-invariant family of $S U$-rank 1 types. Then, either there exists an unbounded type-definable $\Upsilon$-open set over some small set that is almost $\mathcal{P}_{0}$-internal and has bounded finite $S U$-rank, or every complete type $p \in S(A)$ that is internal in $\mathcal{P}_{0}$ is essentially 1-based over $\emptyset$ by means of $\Upsilon$. In particular, either there exists an unbounded type-definable $\Upsilon$-open set that is almost $\mathcal{P}_{0}$-internal and has bounded finite $S U$-rank, or whenever $p \in S(A)$, where $A$ is countable, and $p$ is analyzable in $\mathcal{P}_{0}, p$ is analyzable in $\mathcal{P}_{0}$ by essentially 1-based types by means of $\Upsilon$.

Proof: $\Upsilon$ will be fixed and we'll freely omit the phrase "by means of $\Upsilon$ ". To see the "In particular" part, work over a countable $A$ and assume that every $p^{\prime} \in S\left(A^{\prime}\right)$, with countable $A^{\prime} \supseteq A$, that is internal in $\mathcal{P}_{0}$, is essentially 1-based over $A$. Moreover, assume $p \in S(A)$ is non-algebraic and every nonalgebraic extension of $p$ is non-foreign to $\mathcal{P}_{0}$. Then, for $a \models p$ there exists $a^{\prime} \in d c l(A a) \backslash \operatorname{acl}(A)$ such that $\operatorname{tp}\left(a^{\prime} / A\right)$ is $\mathcal{P}_{0}$-internal and thus essentially 1-based over $A$ by our assumption. Thus, by repeating this process we get that $p$ is analyzable in $\mathcal{P}_{0}$ by essentially 1-based types.

We now prove the main part. Assume there exists $p \in S(A)$ that is internal in $\mathcal{P}_{0}$, and $p$ is not essentially 1-based over $\emptyset$. By the definition, there exist a finite tuple $d$ of realizations of $p$ and $b$ that is independent from $d$ over $A$, and a finite tuple $\bar{c}$ of realizations of types from $\mathcal{P}_{0}$ over $A b$ such that $d \in d c l(A b \bar{c})$, and there exists a type-definable $\Upsilon$-open set $\mathcal{U}$ over $A d$ such that $a$ is independent from $A$ for all $a \in \mathcal{U}$ and $\{a \in \mathcal{U} \mid C b(a / A d) \nsubseteq \operatorname{acl}(a)\}$ is not nowhere dense in the Stone-topology of $\mathcal{U}$. So, since $\Upsilon$ refines the Stone-topology, by intersecting $\mathcal{U}$ with a definable set, we may assume that $\{a \in \mathcal{U} \mid C b(a / A d) \nsubseteq a c l(a)\}$ is dense in the Stone-topology of $\mathcal{U}$. Now, for each (finite) subsequence $\bar{c}_{0}$ of $\bar{c}$, let

$F_{\bar{c}_{0}}=\left\{a \in \mathcal{U} \mid \exists b^{\prime}, \bar{c}_{0}^{\prime}, \bar{c}_{1}^{\prime}\right.$ s.t. $t p\left(b^{\prime} \bar{c}_{0}^{\prime} \bar{c}_{1}^{\prime} / A d\right)=\operatorname{tp}\left(b \bar{c}_{0}\left(\bar{c} \backslash \bar{c}_{0}\right) / A d\right)$ and $\left.a \downarrow A b^{\prime} \bar{c}_{0}^{\prime}\right\}$.

Note that since $d$ is independent from $b$ over $A$, any $a \in \mathcal{U}$ is independent from $A b^{\prime}$ whenever $t p\left(b^{\prime} / A d\right)=t p(b / A d)$ and ${ }^{a} \underset{A d}{\downarrow} b^{\prime}$. Thus $F_{\langle\rangle}=\mathcal{U}$. Let $\bar{c}_{0}^{*}$ be a maximal subsequence (with respect to inclusion) of $\bar{c}$ such that $F_{\bar{c}_{0}^{*}}$ has 
non-empty Stone-interior in $\mathcal{U}$ over $A d$ (note that $F_{\bar{c}}$ has no Stone-interior relatively in $\mathcal{U})$. Let $\mathcal{U}^{*}=\bigcap_{\bar{c}_{0}^{*} \subset \bar{c}^{\prime} \subseteq \bar{c}} \mathcal{U} \backslash F_{\bar{c}^{\prime}}$. Note that each $F_{\bar{c}^{\prime}}$ is Stone closed relatively in $\mathcal{U}$. Thus $\mathcal{U}^{*}$ is Stone-dense and Stone-open in $\mathcal{U}$ and therefore there exists a non-empty relatively Stone-open in $\mathcal{U}$ set $W^{*} \subseteq F_{\bar{c}_{0}^{*}} \cap \mathcal{U}^{*}$. As $\mathcal{U}$ is type-definable, we may assume $W^{*}$ is type-definable.

Claim 4.6 $W^{*}$ is a non-empty $\Upsilon$-open set over Ad such that $\left\{a \in W^{*} \mid C b(a / A d) \nsubseteq\right.$ acl $(a)\}$ is dense in the Stone-topology of $W^{*}$ and for every $a \in W^{*}$ we have: there exists $b^{\prime} \bar{c}_{0}^{\prime} \bar{c}_{1}^{\prime}=t p\left(b \bar{c}_{0}^{*}\left(\bar{c} \backslash \bar{c}_{0}^{*}\right) / A d\right)$ such that $a$ is independent from $A b^{\prime} \bar{c}_{0}^{\prime}$ over $\emptyset$ and moreover, for every $b^{\prime} \bar{c}_{0}^{\prime} \bar{c}_{1}^{\prime} \models t p\left(b \bar{c}_{0}^{*}\left(\bar{c} \backslash \bar{c}_{0}^{*}\right) / A d\right)$ such that a is independent from $A b^{\prime} \bar{c}_{0}^{\prime}$ we necessarily have $\bar{c}_{1}^{\prime} \in \operatorname{acl}\left(a A b^{\prime} \bar{c}_{0}^{\prime}\right)$.

Proof: The first part is immediate by the fact that $W^{*} \subseteq F_{\bar{c}_{0}^{*}}$. For the "moreover" part note that since $a \in W^{*} \subseteq \mathcal{U}^{*}$, we get that by the definition of $\mathcal{U}^{*}, c^{\prime} \in \operatorname{acl}\left(a A b^{\prime} \bar{c}_{0}^{\prime}\right)$ for every $b^{\prime} \bar{c}_{0}^{\prime} \bar{c}_{1}^{\prime} \models t p\left(b \bar{c}_{0}^{*}\left(\bar{c} \backslash \bar{c}_{0}^{*}\right) / A d\right)$ and $c^{\prime} \in \bar{c}_{1}^{\prime}$ (as $S U\left(c^{\prime} / A b^{\prime}\right) \leq 1$ for every $\left.c^{\prime} \in \bar{c}_{1}^{\prime}\right)$.

Let us now define a set $V$ over $A d$ by

$$
\begin{gathered}
V=\left\{\left(e^{\prime}, b^{\prime}, \bar{c}_{0}^{\prime}, \bar{c}_{1}^{\prime}, a^{\prime}\right) \mid \text { if } \operatorname{tp}\left(b^{\prime} \bar{c}_{0}^{\prime} \bar{c}_{1}^{\prime} / A d\right)=\operatorname{tp}\left(b \bar{c}_{0}^{*}\left(\bar{c} \backslash \bar{c}_{0}^{*}\right) / A d\right) \text { and } a^{\prime} \downarrow A b^{\prime} \bar{c}_{0}^{\prime}\right. \\
\text { then } \left.e^{\prime} \in \operatorname{acl}\left(C b\left(A b^{\prime} \bar{c}_{0}^{\prime} \bar{c}_{1}^{\prime} / a^{\prime}\right)\right)\right\} .
\end{gathered}
$$

Let $V^{*}=\left\{e^{\prime} \mid \exists a^{\prime} \in W^{*} \forall b^{\prime}, \bar{c}_{0}^{\prime}, \bar{c}_{1}^{\prime} V\left(e^{\prime}, b^{\prime}, \bar{c}_{0}^{\prime}, \bar{c}_{1}^{\prime}, a^{\prime}\right)\right\}$.

Claim 4.7 $V^{*}$ is a $\Upsilon$-open set over Ad.

Proof: Recall the following fact [S2, Proposition 2.4].

Fact 4.8 Let $q(x, y) \in S(\emptyset)$ and let $\chi(x, y, z)$ be an $\emptyset$-invariant set such that for all $(c, b, a) \models \chi(x, y, z)$ we have $b \unrhd_{a} b c$. Then the set

$$
\mathcal{U}=\{(e, c, b, a) \mid e \in \operatorname{acl}(C b(c b / a))\}
$$

is relatively Stone-open inside the set

$$
F=\{(e, c, b, a) \mid b \downarrow a, \models \chi(c, b, a), t p(c b)=q\} .
$$

(where e is taken from a fixed sort too). 
By Fact 4.8 and Claim 4.6, there exists a Stone-open set $V^{\prime}$ over Ad such that for all $a^{\prime} \in W^{*}$ and for all $e^{\prime}, b^{\prime}, \bar{c}_{0}^{\prime}, \bar{c}_{1}^{\prime}$ we have $V^{\prime}\left(e^{\prime}, b^{\prime}, \bar{c}_{0}^{\prime}, \bar{c}_{1}^{\prime}, a^{\prime}\right)$ if and only if $V\left(e^{\prime}, b^{\prime}, \bar{c}_{0}^{\prime}, \bar{c}_{1}^{\prime}, a^{\prime}\right)$. Thus, we may replace $V$ by $V^{\prime}$ in the definition of $V^{*}$. As Stone-open sets are closed under the $\forall$ quantifier, the $\Upsilon$ topology refines the Stone-topology and closed under product by a full Stone-space and closed under projections, we conclude that $V^{*}$ is a $\Upsilon$-open set.

Claim 4.9 For appropriate sort for $e^{\prime}$, the set $V^{*}$ is unbounded and is almost $\mathcal{P}_{0}$-internal (over $A d$ ) and thus has finite $S U$-rank over Ad.

Proof: First, note the following general observation.

Fact 4.10 Assume $d \in d c l(c)$. Then $C b(d / a) \in d c l(C b(c / a))$ for all a.

Let $a^{*} \in W^{*}$ be such that $C b\left(a^{*} / A d\right) \nsubseteq \operatorname{acl}\left(a^{*}\right)$. Then $C b\left(A d / a^{*}\right) \nsubseteq \operatorname{acl}(A d)$. By Fact 4.10, there exists $e^{*} \notin \operatorname{acl}(A d)$ such that $e^{*} \in \operatorname{acl}\left(C b\left(A b^{\prime} \bar{c}_{0}^{\prime} \bar{c}_{1}^{\prime} / a^{*}\right)\right)$ for all $b^{\prime} \bar{c}_{0}^{\prime} \bar{c}_{1}^{\prime} \models t p\left(b \bar{c}_{0}^{*}\left(\bar{c} \backslash \bar{c}_{0}^{*}\right) / A d\right)$. In particular, $e^{*} \in V^{*}$. Thus, if we fix the sort for $e^{\prime}$ in the definition of $V^{*}$ to be the sort of $e^{*}$, then $V^{*}$ is unbounded. Now, let $e^{\prime} \in V^{*}$. Then for some $a^{\prime} \in W^{*}, \models V\left(e^{\prime}, \bar{c}_{0}^{\prime}, \bar{c}_{1}^{\prime}, b^{\prime}, a^{\prime}\right)$ for all $b^{\prime}, \bar{c}_{0}^{\prime}, \bar{c}_{1}^{\prime}$. By Claim 4.6, there exists $b^{\prime} \bar{c}_{0}^{\prime} \bar{c}_{1}^{\prime} \models t p\left(b \bar{c}_{0}^{*}\left(\bar{c} \backslash \bar{c}_{0}^{*}\right) / A d\right)$ such that $a^{\prime}$ is independent from $A b^{\prime} \bar{c}_{0}^{\prime}$ over $\emptyset$. Thus, by the definition of $V^{*}$ and $V$, $e^{\prime} \in \operatorname{acl}\left(C b\left(A b^{\prime} \bar{c}_{0}^{\prime} \bar{c}_{1}^{\prime} / a^{\prime}\right)\right)$. Since $A b^{\prime}$ is independent from $a^{\prime}$ over $\emptyset, t p\left(e^{\prime}\right)$ is almost $\mathcal{P}_{0}$-internal (as $C b\left(A b^{\prime} \bar{c}_{0}^{\prime} \bar{c}_{1}^{\prime} / a^{\prime}\right)$ is in the definable closure of any Morley sequence of $\left.\operatorname{Lstp}\left(A b^{\prime} \bar{c}_{0}^{\prime} \bar{c}_{1}^{\prime} / a^{\prime}\right)\right)$. In particular, $t p\left(e^{\prime} / A d\right)$ is almost $\mathcal{P}_{0}$-internal by Fact 2.1 and therefore $t p\left(e^{\prime} / A d\right)$ has finite $S U$-rank.

Claim 4.11 There exists $V^{* *} \subseteq V^{*}$ that is unbounded, type-definable and $\Upsilon$-open over Ad.

Proof: By the definition of $V^{*}$ and the proof of Claim 4.7 there exist a Stone open set $V_{0}$ over $A d$ such that $V^{*}=\left\{e^{\prime} \mid \exists a^{\prime} \in W^{*}\left(V_{0}\left(e^{\prime}, a^{\prime}\right)\right)\right\}$. By replacing $V_{0}$ by a definable set and using the fact that $W^{*}$ is type-definable and that $\Upsilon$ is a projection-closed family of topologies we get the required set $V^{* *}$

Now, by the proof of Claim 4.9 we know that for all $e^{\prime} \in V^{* *}$ we have $e^{\prime} \in \operatorname{acl}\left(C b\left(A b^{\prime} \bar{c}_{0}^{\prime} \bar{c}_{1}^{\prime} / a^{\prime}\right)\right)$ for some $a^{\prime} \in W^{*}$ and some $b^{\prime}, \bar{c}_{0}^{\prime}, \bar{c}_{1}^{\prime}$ such that $a^{\prime}$ is independent from $A b^{\prime} \bar{c}_{0}^{\prime}$ over $\emptyset$ and $b^{\prime} \bar{c}_{0}^{\prime} \bar{c}_{1}^{\prime} \models t p\left(b \bar{c}_{0}^{*}\left(\bar{c} \backslash \bar{c}_{0}^{*}\right) / A d\right)$. Let $q=t p(A b)$. 
For every $\chi=\chi\left(x, y_{0}, \ldots, y_{n}, \bar{z}_{0}, \bar{z}_{1}, \ldots \bar{z}_{n}\right) \in L$ (for some $n<\omega$ ) such that $\forall y_{0} y_{1} \ldots y_{n} \bar{z}_{0} \bar{z}_{1} \ldots \bar{z}_{n} \exists^{<\infty} x \chi\left(x, y_{0}, y_{1}, \ldots y_{n}, \bar{z}_{0}, \bar{z}_{1}, \ldots \bar{z}_{n}\right)$, let

$$
\begin{aligned}
F_{\chi}=\left\{e \in V^{* *} \mid\right. & \models \chi\left(e, C_{0}, C_{1}, . . C_{n}, \bar{c}_{0}, \bar{c}_{1}, \ldots \bar{c}_{n}\right) \text { for some } \bar{c}_{0}, \ldots \bar{c}_{n} \text { and some } \\
& \emptyset \text { - independent sequence }\left(C_{i} \mid i \leq n\right) \text { of realization of } q \\
& \text { such that } \left.\operatorname{tp}\left(C_{i}, \bar{c}_{i}\right)=\operatorname{tp}(A b, \bar{c}) \text { and } e \downarrow\left(C_{i} \mid i \leq n\right)\right\} .
\end{aligned}
$$

Note that eaxh $F_{\chi}$ is type-definable. By the aforementioned, we get that $V^{* *} \subseteq \bigcup_{\chi} F_{\chi}$ (the union is over each $\chi$ as above). By the Baire category theorem applied to the Stone-topology of the Stone-closed set $V^{* *} \backslash a c l(A d)$, there exists $\theta \in L(A d)$ such that

$$
\tilde{V} \equiv \theta^{\mathcal{C}} \cap\left(V^{* *} \backslash \operatorname{acl}(A d)\right) \neq \emptyset \text { and } \tilde{V} \subseteq F_{\chi^{*}}
$$

for some $\chi^{*}$ as above. Clearly, $\tilde{V}$ is unbounded, type-definable and $\Upsilon$-open (by the assumptions on $\Upsilon$ ). Now, there exists a fixed $m^{*}<\omega$ such that for every $a \in \tilde{V}, S U(a / A d) \leq m^{*}$ and $t p(a / A d)$ is almost $\mathcal{P}_{0}$-internal (as $t p(a)$ is almost $\mathcal{P}_{0}$-internal). This completes the proof of the main part of the theorem.

The proof of the main result of this section now follows exactly in the same way as in [S2]. We write the proof for completeness.

Proof of Theorem 4.3 Our assumptions are clearly a special case of the assumptions of Theorem 4.5, thus we only need to prove the first part. By the conclusion of Theorem 4.5, we may assume that there exists a $\tau^{f}$-open set $\mathcal{U}$ of bounded finite $S U$-rank over some small set $A$ that is almost $\mathcal{P}_{0^{-}}$ internal. By Fact 2.10, there exists exists a weakly-minimal $\theta(x, b) \in L(B)$ for some small set $B \supseteq A$, such that $\theta^{\mathcal{C}} \subseteq \mathcal{U} \cup \operatorname{acl}(B)$. Now, $t p(a / B)$ is almost $\mathcal{P}_{0}$-internal for every $a \in \theta^{\mathcal{C}}$, and so $t p(a / b)$ ( $b$ is the parameter of $\left.\theta(x, b)\right)$ is almost $\mathcal{P}_{0}$-internal for every $a \in \theta^{\mathcal{C}}$ (by taking non-forking extensions).

\section{Main result}

From now on we assume $T$ is an arbitrary simple theory with elimination of imaginaries unless stated otherwise. We work in $\mathcal{C}$. 
Definition 5.1 1) We say that $T$ is analyzable in $S U$-rank 1 types if every type is analyzable in the family of $S U$-rank 1 types.

2) We say that $T$ is coordinatized in finite rank if for every $a \in \mathcal{C}$ and $A \subseteq \mathcal{C}$ such that $a \notin \operatorname{acl}(A)$ there exists $a^{\prime} \in \operatorname{acl}(a A) \backslash \operatorname{acl}(A)$ with $S U\left(a^{\prime} / A\right)<\omega$.

Lemma 5.2 Assume $T$ is hypersimple. $T$ is coordinatized in finite rank iff $T$ is analyzable in $S U$-rank 1 types.

Proof: If $T$ is analyzable in $S U$-rank 1 types then clearly $T$ is coordinatized in finite rank. Assume now that $T$ is coordinatized in finite rank. We first note the following.

Claim 5.3 Let $T$ be any simple theory. Let $a \in \mathcal{C}$ be such that $S U(a)=n<$ $\omega$ and such that for some $b \in \mathcal{C}$ with $S U(b)<\infty$ we have $S U(a / b)=n-1$. Then $\operatorname{tp}(a)$ is non-orthogonal to an SU-rank 1 hyperimaginary type.

Proof: Let $e=C b(\operatorname{Lstp}(a / b))$ (e is a hyperimaginary). Since $S U(e)<\infty$ (as we assume $S U(b)<\infty$ ), there exists a set $A$ such that $S U(e / A)=1$. By extension we may clearly assume $e_{e}^{\downarrow} A$. We claim that $e \in \operatorname{bdd}(a A)\left(^{*}\right)$. Indeed, otherwise $e \underset{A}{\downarrow} a$ and so $e \in b d d(A)$ (as $t p(a / e)$ is canonical), a contradiction to $S U(e / A)=1$. Now, $S U(a / e A)=S U(a / e)=n-1$. By $\left(^{*}\right)$, $S U(a / A)=S U(a e / A) \geq S U(a / e A)+S U(e / A)=n$. Thus $a \downarrow A$ and so $\operatorname{tp}(a)$ is non-orthogonal to $t p(e / A)$.

Now, let $a, A$ be given such that $a \notin a c l(A)$. By our assumption, there exists $a^{\prime} \in \operatorname{acl}(a A) \backslash \operatorname{acl}(A)$ with $S U\left(a^{\prime} / A\right)=n$ for some $n<\omega$. Let $b \in \mathcal{C}$ be such that $S U(a / A b)=n-1$ and let $\left(b_{i} \mid i<\alpha\right)$ be such that $b_{i} \in \operatorname{acl}(b A)$ and $0<S U\left(b_{i} / A b_{<i}\right)<\omega$ for all $i<\alpha$ and such that $\operatorname{acl}(A b)=\operatorname{acl}\left(A \cup\left\{b_{i} \mid i<\right.\right.$ $\alpha\})$. As $a_{A}^{\prime} \varliminf^{\lfloor}$, there exists a minimal $i^{*}<\alpha$ such that $a^{\prime} \underset{A}{\lfloor}\left\{b_{i} \mid i \leq i^{*}\right\}$. Then $a^{\prime} \underset{A}{\downarrow}\left\{b_{i} \mid i<i^{*}\right\}$. Now, $a^{\prime}$ satisfies the assumptions of Claim 5.3 when working over $A \cup\left\{b_{i} \mid i<i^{*}\right\}$. Thus $\operatorname{tp}\left(a^{\prime} / A \cup\left\{b_{i} \mid i<i^{*}\right\}\right)$ is nonorthogonal to an $S U$-rank 1 type which we may clearly assume to be a type of an imaginary. Thus $t p\left(a^{\prime} / A\right)$ is non-orthogonal an $S U$-rank 1 imaginary type and so is $\operatorname{tp}(a / A)$ (as $\left.a^{\prime} \in \operatorname{acl}(a A)\right)$. 
We start with the following proposition that generalizes the main result in [S1]; the proof is similar but applies Theorem 4.3.

Proposition 5.4 Assume $T$ is a countable hypersimple theory that is coordinatized in finite rank and eliminates $\exists^{\infty}$. Then there exists a weakly minimal formula.

Proof: By Lemma 5.2, $T$ is analyzable in SU-rank 1 types. As $T$ eliminates $\exists^{\infty}, T$ is EPFO by Fact 2.11. By Fact 2.9, $T$ is PCFT. Let $\mathcal{P}_{0}$ be the family of all SU-rank 1 types. By Theorem 4.3 , we may assume that every complete finitary type over a countable set is analyzable in $\mathcal{P}_{0}$ by essentially 1-based types by means of $\tau^{f}$. We recall the following fact that for simplicity we state for a special case [S3, Corollary 4.5]. Infact, the result is valid for a large class of sets $\left(\tilde{\tau}_{\text {low }}^{f}\right.$-sets) instead of the specific set $\mathcal{U}_{0}$ that is fixed in the following statement.

Fact 5.5 Let $T$ be a countable simple theory with EPFO. Let $\mathcal{U}_{0}=\mathcal{C}^{s} \backslash$ acl $(\emptyset)$ for some non-algebraic sort $s$ say. Assume for every $a \in \mathcal{U}_{0}$ there exists $a^{\prime} \in \operatorname{acl}(a) \backslash \operatorname{acl}(\emptyset)$ such that $S U_{\text {se }}\left(a^{\prime}\right)<\omega$. Then there exists an unbounded $\tau_{\infty}^{f}$-open set $\mathcal{U}$ over a finite set such that $\mathcal{U}$ has bounded finite $S U_{\text {se-rank. }}$

$T$ satisfies the assumptions of Fact 5.5 , so let $\mathcal{U}$ be a set as in its conclusion. In particular, $S U_{s}(\mathcal{U})=n$ for some $n<\omega$. Recall now the following easy lemma.

Lemma 5.6 [S1, Lemma 7.4] Assume $\mathcal{U}$ is an unbounded $\tau_{\infty}^{f}$-open set of bounded finite $S U_{s}$-rank over some finite set $A$. Then there exists a $\tau_{\infty}^{f}$-open set $\mathcal{U}^{*} \subseteq \mathcal{U}$ over some finite set $B^{*} \supseteq A$ of $S U_{s}$-rank 1 .

By Lemma 5.6, we may assume $S U_{s}(\mathcal{U})=1, \mathcal{U}$ is a type-definable $\tau^{f}$-open set over a finite set $A_{0}$. We claim $S U(\mathcal{U})=1$. Indeed, otherwise there exists $a$ and $d \in \mathcal{U}$ such that $d \underset{A_{0}}{\not{X}} a$ and $d \notin \operatorname{acl}\left(a A_{0}\right)$. Since every finitary type over a countable set is analyzable in $\mathcal{P}_{0}$, there exists $\left(a_{i} \mid i \leq\right.$ $\alpha) \subseteq d c l\left(a A_{0}\right)$ with $a_{\alpha}=a$ (where $\left.\alpha<\omega_{1}\right)$ such that $\operatorname{tp}\left(a_{i} / A_{0} \cup\left\{a_{j} \mid j<i\right\}\right)$ is essentially 1 -based over $A_{0}$ by means of $\tau^{f}$ for all $i \leq \alpha$. Now, let $i^{*} \leq \alpha$ be minimal such that there exists $d^{\prime} \in \mathcal{U}$ satisfying $d^{\prime} \underset{A_{0}}{\not}\left\{a_{i} \mid i \leq i^{*}\right\}$ and 
$d^{\prime} \notin \operatorname{acl}\left(A_{0} \cup\left\{a_{i} \mid i \leq i^{*}\right\}\right)$. Pick $\phi\left(x, a^{\prime}\right) \in L\left(A_{0} \cup\left\{a_{i} \mid i \leq i^{*}\right\}\right)$ that forks over $A_{0}$ and such that $\phi\left(d^{\prime}, a^{\prime}\right)$. Let

$$
V=\left\{d \in \mathcal{U} \mid \phi\left(d, a^{\prime}\right) \text { and } d \notin \operatorname{acl}\left(A_{0} \cup\left\{a_{i} \mid i \leq i^{*}\right\}\right)\right\}
$$

By minimality of $i^{*}, d$ is independent from $\left\{a_{i} \mid i<i^{*}\right\}$ over $A_{0}$ for all $d \in$ $V$. Clearly $V$ is type-definable and by Fact [2.6, $V$ is a $\tau^{f}$-open set over $A_{0} \cup\left\{a_{i} \mid i \leq i^{*}\right\}$. Now, since $\operatorname{tp}\left(a_{i^{*}} / A_{0} \cup\left\{a_{i} \mid i<i^{*}\right\}\right)$ is essentially 1-based over $A_{0}$ by means of $\tau^{f}$, the set

$$
\left\{d \in V \mid C b\left(d / A_{0} \cup\left\{a_{i} \mid i \leq i^{*}\right\}\right) \in b d d\left(d A_{0}\right)\right\}
$$

contains a relatively Stone-open and Stone-dense subset of $V$. In particular, there exists $d^{*} \in V$ such that $\operatorname{tp}\left(d^{*} / A_{0} \cup\left\{a_{i} \mid i \leq i^{*}\right\}\right.$ doesn't fork over $\operatorname{acl}\left(A_{0} d^{*}\right) \cap \operatorname{acl}\left(A_{0} \cup\left\{a_{i} \mid i \leq i^{*}\right\}\right)$. Since we know $d^{*} \underset{A_{0}}{\not} A_{0} \cup\left\{a_{i} \mid i \leq i^{*}\right\}$, Fact2.16]implies $d^{*} \underset{A_{0}}{\not A_{s}} A_{0} \cup\left\{a_{i} \mid i \leq i^{*}\right\}$. Hence $d^{*} \in V$ implies $S U_{s}\left(d^{*} / A_{0}\right) \geq$ 2, which contradicts $S U_{s}(\mathcal{U})=1$. Thus we have proved $S U(\mathcal{U})=1$. Now, by Fact 2.10 there exists a definable set of $S U$-rank 1.

Before stating the main theorem, we give some terminology and easy remarks. Recall that we work in $\mathcal{C}=\mathcal{C}^{e q}$ and that $\left(\mathcal{R}_{\mathcal{C}}^{\lambda}, \leq_{\mathcal{C}}\right)$ is the partial order of reducts of $\mathcal{C}$ of size $\leq \lambda$.

Definition 5.7 Let $\mathcal{C} \mid L^{-} \in \mathcal{R}_{\mathcal{C}}^{\lambda}$. We will say that $\mathcal{C} \mid L^{-}$is eq-closed if $T^{-}=$ $T h\left(\mathcal{C} \mid L^{-}\right)$has uniform elimination of imaginaries, i.e. for every definable equivalence relation $E \in L^{-}$on $S_{0} \times S_{1} \times \ldots S_{k}$, where $S_{i}$ are sorts of $L^{-}$, there is a definable function $f_{E} \in L^{-}$whose domain is $\left(S_{0} \times S_{1} \times \ldots S_{k}\right)^{\mathcal{C}}$ such that for all $\bar{a}, \bar{b}$, we have $f_{E}(\bar{a})=f_{E}(\bar{b})$ iff $E(\bar{a}, \bar{b})$.

Remark 5.8 For every reduct $\mathcal{C} \mid L^{-} \in \mathcal{R}_{\mathcal{C}}^{\lambda}$ there exists a reduct $\mathcal{C} \mid L^{*} \in \mathcal{R}_{\mathcal{C}}^{\lambda}$ that is eq-closed and is an expansion of $\mathcal{C} \mid L^{-}$. Thus for every infinite cardinal $\lambda$, the set of reducts in $\mathcal{R}_{\mathcal{C}}^{\lambda}$ that are eq-closed is a club in $\left(\mathcal{R}_{\mathcal{C}}^{\lambda}, \leq_{\mathcal{C}}\right)$.

Proof: Expand the reduct $\mathcal{C} \mid L^{-}$of $\mathcal{C}=\mathcal{C}^{e q}$ by adding for every definable equivalence relation $E$ on $S_{0} \times S_{1} \times \ldots S_{k}$, where $S_{i}$ are sorts of $L^{-}$and $E \in L^{-}$, a definable function $f_{E} \in L$ whose domain is $\left(S_{0} \times S_{1} \times \ldots S_{k}\right)^{\mathcal{C}}$ and is onto the interpretation of some sort of $L$ such that $f_{E}(\bar{a})=f_{E}(\bar{b})$ iff $E(\bar{a}, \bar{b})$. Now, 
the resulting expansion will have uniform elimination of imaginaries. It is immediate that the set of eq-closed reducts in $\mathcal{R}_{\mathcal{C}}^{\lambda}$ is closed in $\left(\mathcal{R}_{\mathcal{C}}^{\lambda}, \leq_{\mathcal{C}}\right)$.

Now, note the following easy general remark on clubs.

Remark 5.9 Let $(P, \leq)$ be a directed partial order that is $\omega$-closed (i.e. any increasing sequence $\left(a_{i} \mid i<\omega\right)$ has a supremum). Then the intersection of finitely many clubs in $(P, \leq)$ is a club.

In the proof we will refer to the following notion.

Definition 5.10 We say that $T$ is strongly non-supersimple if $D(\phi(x, a))=$ $\infty$ for every non-algebraic $\phi(x, a) \in L(\mathcal{C})$.

Remark 5.11 Note that $T$ is strongly non-supersimple iff for every nonalgebraic $\phi(x, a) \in L(\mathcal{C})$ there exists a non-algebraic $\psi(x, b) \in L(\mathcal{C})$ such that $\psi(x, b) \vdash \phi(x, a)$ and $\psi(x, b)$ forks over $a$ iff there does not exist a weakly minimal formula.

Theorem 5.12 Let $T=T^{e q}$ be a hypersimple unidimensional theory. Assume there is a club of countable reducts of $T$ in $\left(\mathcal{R}_{\mathcal{C}}^{\aleph_{0}}, \leq_{\mathcal{C}}\right)$ that are coordinatized in finite rank. Then $T$ is supersimple.

Proof: First, if $T$ is not strongly non-supersimple then we are done by Fact 2.2. Therefore, we may assume $T$ is strongly non-supersimple. By Fact 2.3, $T$ eliminates $\exists^{\infty}$, thus every reduct of $T$ eliminates $\exists^{\infty}$.

Claim 5.13 The set $\tilde{\mathcal{C}}_{1}$ of countable strongly non-supersimple reducts of $\mathcal{C}$ is a club in $\left(\mathcal{R}_{\mathcal{C}}^{\aleph_{0}}, \leq_{\mathcal{C}}\right)$.

Proof: First, we prove that $\tilde{\mathcal{C}}_{1}$ is unbounded in $\left(\mathcal{R}_{\mathcal{C}}^{\aleph_{0}}, \leq_{\mathcal{C}}\right)$. Let $\mathcal{C} \mid L^{-} \in$ $\mathcal{R}_{\mathcal{C}}^{\aleph_{0}}$. We construct by induction an increasing sequence of reducts $\left(\mathcal{C}_{n} \mid n<\right.$ $\omega), \mathcal{C}_{n} \in \mathcal{R}^{\aleph_{0}}$, where $\mathcal{C}_{n}=\mathcal{C} \mid L_{n}^{-}$for some countable sublanguage $L_{n}^{-}$of $L$, $T_{n}^{-}=T h\left(\mathcal{C}_{n}\right)$ in the following way. Let $\mathcal{C}_{0}=\mathcal{C} \mid L_{0}^{-}, L_{0}^{-}=L^{-}$and assume $L_{k}^{-}$have already been defined for $k \leq n$. We define $L_{n+1}^{-}$. For any fixed $\phi(x, y) \in L_{n}^{-} \backslash L_{n-1}^{-}$we define a finite set of formulas $\Delta_{\phi}=\left\{\psi_{i} \mid i \leq n(\phi)\right\}$, where $\psi_{i}=\psi_{i}\left(x, y_{i}\right) \in L=L(T), n(\phi)<\omega$ in the following way. Since $T$ is strongly non-supersimple, for every $a \in \mathcal{C}$ such that $\exists^{\infty} x \phi(x, a)$, there exists 
$\psi_{a}(x, z) \in L$ and some $b \in \mathcal{C}$ such that $\psi_{a}(x, b) \vdash \phi(x, a), \psi_{a}(x, b)$ forks over $a$ and $\exists^{\infty} x \psi_{a}(x, b)(*)$. For every $\psi(x, z) \in L$ let

$$
\theta_{\phi, \psi}(z, y)=\exists^{\infty} x \psi(x, z) \wedge \forall x(\psi(x, z) \rightarrow \phi(x, y)) .
$$

By elimination of $\exists^{\infty}$ (in $\mathcal{C}$ ), $\theta_{\phi, \psi}(z, y)$ is definable. Now, let $Q_{\psi, \theta_{\phi, \psi}}(y)$ be the relation in Fact 2.8 defined for $\theta_{\phi, \psi}, \psi$. So, for every $a \in \mathcal{C}, \neg Q_{\psi, \theta_{\phi, \psi}}(a)$ iff there exists $b$ such that $\psi(x, b)$ is not algebraic, $\psi(x, b) \vdash \phi(x, a)$ and $\psi(x, b)$ forks over $a$. Since $T$ is EPFO, we know that each $\neg Q_{\psi, \theta_{\phi, \psi}}$ is Stone-open. By $\left.{ }^{*}\right)$, in $\mathcal{C}$ :

$$
\exists^{\infty} x \phi(x, y) \vdash \bigvee_{\psi \in L} \neg Q_{\psi, \theta_{\phi, \psi}}(y) .
$$

By compactness, there are $\psi_{0}=\psi_{0}(\phi), \ldots, \psi_{n(\phi)}=\psi_{n(\phi)}(\phi) \in L$ such that in $\mathcal{C}:$

$$
\exists^{\infty} x \phi(x, y) \vdash \bigvee_{i<n(\phi)} \neg Q_{\psi_{i}, \theta_{\phi, \psi_{i}}}(y) \quad(* *) .
$$

Now, let $\Delta_{\phi}=\left\{\psi_{i}(\phi) \mid i \leq n(\phi)\right\}$. Let $L_{n+1}^{-}$be the set of formulas generated by the set

$$
\nu_{n+1}=L_{n}^{-} \cup \bigcup\left\{\Delta_{\phi} \mid \phi(x, y) \in L_{n}^{-} \backslash L_{n-1}^{-}\right\},
$$

where the set of sorts attached to $L_{n+1}^{-}$is the set of all sorts of variables that appears in $\nu_{n+1}$. Let $\mathcal{C}_{n+1}=\mathcal{C} \mid L_{n+1}^{-}$. Now, let $L_{\omega}^{-}=\bigcup_{n<\omega} L_{n}^{-}$and let $\mathcal{C}_{\omega}=\mathcal{C} \mid L_{\omega}^{-}, T_{\omega}^{-}=T h\left(\mathcal{C}_{\omega}\right)$. We claim that $T_{\omega}^{-}$is strongly non-supersimple. Indeed, given a formula $\phi_{\omega}(x, y) \in L_{\omega}^{-}$, let $a \in \mathcal{C}_{\omega}$ be such that $\models \exists^{\infty} x \phi_{\omega}(x, a)$. Then, by $\left({ }^{*}\right)$ there exists $\psi(x, z) \in \Delta_{\phi_{\omega}} \subseteq L_{\omega}^{-}$such that for some $b$ we have $\psi(x, b) \vdash \phi_{\omega}(x, a)$ and $\psi(x, b)$ forks over $a$ in $\mathcal{C}$ and thus in particular $\psi(x, b)$ forks over $a$ in $\mathcal{C}_{\omega}$. Thus $T_{\omega}^{-}$is strongly non-supersimple. Now, to show that $\tilde{\mathcal{C}}_{1}$ is closed in $\left(\mathcal{R}_{\mathcal{C}}^{\aleph_{0}}, \leq_{\mathcal{C}}\right)$, let $\tilde{\mathcal{C}}$ be a chain in $\tilde{\mathcal{C}}_{1}$. We claim that $\mathcal{C}^{*}=\bigcup \tilde{\mathcal{C}}$ (the universe of $\mathcal{C}^{*}$ is the union of the interpretations of the sorts of all members of $\tilde{\mathcal{C}}$ and likewise for the definable sets of $\left.\mathcal{C}^{*}\right)$ is strongly non-supersimple. Indeed, let $\phi(x, a) \in L\left(\mathcal{C}^{*}\right)$ be non-algebraic. Then there exists $\mathcal{C}_{0}=\mathcal{C} \mid L_{0} \in \tilde{\mathcal{C}}$ for some countable sublanguage $L_{0}$ of $L$ such that $\phi(x, a) \in L_{0}\left(\mathcal{C}_{0}\right)$. Since $\operatorname{Th}\left(\mathcal{C}_{0}\right)$ is strongly non-supersimple, there exists a non-algebraic $\psi(x, b) \in L_{0}\left(\mathcal{C}_{0}\right)$ such that $\psi(x, b) \vdash \phi(x, a)$ and $\psi(x, b)$ forks over $a$ in $\mathcal{C}_{0}$. By Ramsey and compactness, there exists a formula $\psi\left(x, b^{\prime}\right)$ that is $a$-conjuagate to $\psi(x, b)$ in $\mathcal{C}_{0}$ and that forks over $a$ in the sense of $\mathcal{C}^{*}$. Thus $T h\left(\mathcal{C}^{*}\right)$ is strongly non-supersimple. 
By Claim 5.13, Claim 3.3, Remark 5.8, the assumptions of the theorem and Remark [5.9, there is a club of reducts in $\mathcal{R}_{\mathcal{C}}^{\aleph_{0}}$ that are strongly nonsupersimple, hypersimple, eq-closed and coordinatized in finite rank. Any such reduct contradicts Proposition 5.4 (as elimination of $\exists^{\infty}$ is clearly preserved under reducts).

\section{References}

[BPV] I.Ben-Yaacov, A.Pillay, E.Vassiliev, Lovely pairs of models, Annals of Pure and Applied Logic 122 (2003), no. 1-3.

[H0] E.Hrushovski, Countable unidimensional stable theories are superstable, unpublished paper.

[H1] E.Hrushovski, Unidimensional theories are superstable, Annals of Pure and Applied Logic, 50 (1990), pgs 117-138.

[HKP] B.Hart, B.Kim and A.Pillay, Coordinatization and canonical bases in simple theories, Journal of Symbolic Logic, 65 (2000), pgs 293-309.

[HP] E. Hrushovski, A. Pillay, Groups definable in local fields and pseudofinite fields, Israel J. Math. 85 (1994), 203 - 262.

[K1] B.Kim, Forking in simple unstable theories, Journal of London Math. Society, 57 (1998), pgs 257-267.

[KP] B.Kim and A.Pillay, Simple theories, Annals of Pure and Applied Logic, 88, 1997 pgs 149-164.

[P] A.Pillay, On countable simple unidimensional theories, Journal of Symbolic Logic 68 (2003), no. 4.

[P1] A.Pillay, The extension property is first order in unidimensional simple theories, unpublished note.

[S0] Z.Shami, On analyzability in the forking topology for simple theories, Annals of Pure Applied Logic 142 (2006), no. 1-3, 115-124.

[S1] Z.Shami, Countable hypersimple unidimensional theories, J. London Math. Soc. Volume 83, Issue 2 (2011), pgs. 309-332. 
[S2] Z.Shami, On uncountable hypersimple unidimensional theories, Arch. Math. Logic 53 (2014), no. 1-2, 203-210.

[S3] Z.Shami, A model theoretic Baire category theorem for simple theories, Fundamenta Mathematicae 220 (2013), no. 3, 191-206.

[W] Frank O. Wagner, Simple Theories, Academic Publishers, Dordrecht, The Netherlands, 2000.

Ziv Shami, E-mail address: zivsh@ariel.ac.il. 